\title{
The central engines of radio-loud quasars
}

\author{
J.-M. Wang ${ }^{1,2,3}$, L. C. $\mathrm{Ho}^{4}$, and R. Staubert ${ }^{1}$ \\ ${ }^{1}$ Institut für Astronomie und Astrophysik, Abt. Astronomie, Universität Tübingen, Sand 1, 72076 Tübingen, Germany \\ ${ }^{2}$ Laboratory for High Energy Astrophysics, Institute of High Energy Physics, CAS, Beijing 100039, PR China \\ 3 Alexander von Humboldt Fellow \\ 4 The Observatories of the Carnegie Institution of Washington, 813 Santa Barbara Street, Pasadena, CA 91101-1292, USA \\ e-mail: 1ho@ociw.edu
}

Received 7 October 2002 / Accepted 29 July 2003

\begin{abstract}
We have assembled a sample of 37 radio-loud quasars that have been imaged with the Hubble Space Telescope in order to investigate their black hole masses, accretion rates, and the structure of their accretion disks. The black hole masses were estimated from the luminosities of the host galaxies, and the accretion powers were extrapolated from the emission-line luminosities. The majority of the quasars have masses in the range $M_{\mathrm{BH}} \approx 10^{8}-10^{9} M_{\odot}$. Their accretion rates, $\dot{M} \approx 0.01-1$ times the Eddington rate, suggest that most of the objects possess standard optically thick, geometrically thin accretion disks, in some cases perhaps accompanied by an optically thin advection-dominated component. The coexistence of strong radio emission and a standard disk conflicts with recent models for jet formation. We discuss modifications of the standard model that can resolve this discrepancy. We find there is a strong correlation between the accretion rate and the extended radio luminosity. This lends support to the idea that the extended radio emission is somehow linked to the accretion disk. Lastly, we combine the present sample of radio-loud quasars with the sample of BL Lac objects studied by Wang et al. (2002) to reevaluate the unification picture for radio-loud active galactic nuclei. Consistent with current ideas for the unification of radio-loud sources, we find that flat-spectrum radio quasars and FR II radio galaxies indeed seem to belong to the same population, as do BL Lac objects and FR I radio galaxies on the opposite end of the luminosity spectrum. However, some members of the low frequencypeaked BL Lac objects may be more closely associated with FR II rather than FR I radio galaxies. We describe how the various subclasses of radio-loud sources can be viewed as a continuous sequence of varying accretion rate.
\end{abstract}

Key words. BL Lacertae objects: general - galaxies: active - galaxies: jets - galaxies: Seyfert - quasars: general

\section{Introduction}

According to the radio-loudness criterion proposed by Kellermann et al. (1989), quasars whose ratio of radio (5 GHz) to optical ( $B$ band) flux exceeds 10 comprise a special population of radio-loud objects (hereafter RLQs). As reviewed by Urry \& Padovani (1995), the powerful radio emission is thought to originate from relativistic jets expelled by the accretion disk around a central supermassive black hole (BH). One of the unsolved problems in astrophysics is the exact physical relation between the jet and the accretion disk. Two observational approaches have been taken to investigate this relation in RLQs and active galactic nuclei (AGNs). On large (kpc to Mpc) scales the relation between the jet radio/kinetic luminosity and the narrow-line luminosity has been examined by many authors (e.g., Baum \& Heckman 1989a,b; Rawlings \& Saunders 1991). It has been found that the narrow-line luminosity is correlated with the kinetic luminosity from the radio lobes. On smaller scales, Celotti \& Fabian (1993) and Celotti et al. (1997) used radio data from very long-baseline

Send offprint requests to: J.-M. Wang,

e-mail: wang@astro.uni-tuebingen.de interferometry to estimate the kinetic luminosities in a large sample of radio-loud objects. Both attempts reached the same conclusion: the relativistic jet is linked with the accretion disk. A more direct connection between the jet and the accretion disk has been found in the RLQ 3C 120 (Marscher et al. 2002). However, how a relativistic jet actually forms from the accretion disk remains an outstanding puzzle (Meier 2001; Blandford 2002).

The situation in Galactic BH candidates suggests that the formation of the jet is somehow linked with the accretion rate. The radio emission from these objects becomes quenched when the system is in a soft (high accretion rate) state, as seen in GX 339-4 (Fender et al. 1999), GRS 1915+105 (Harmon et al. 1997; Fender et al. 1999), GRO J16655-40 (Harmon et al. 1995), Cynus X-3 (McCollough et al. 1999), and Cygnus X-1 (Brocksopp et al. 1999). These objects may be instructive for the case of AGNs, although we should be cautious that some of their properties do not scale linearly with BH mass. The relation between the "states" of the accretion disk and jet production in supermassive BH accretion systems is very poorly understood. 
Table 1. The sample of radio-loud quasars.

\begin{tabular}{|c|c|c|c|c|c|c|c|c|c|}
\hline $\begin{array}{l}\text { No. } \\
\text { (1) }\end{array}$ & $\begin{array}{c}\text { Object } \\
(2)\end{array}$ & $\begin{array}{c}\text { Name } \\
(3)\end{array}$ & $\begin{array}{l}\text { Type } \\
\text { (4) }\end{array}$ & $\begin{array}{c}z \\
(5)\end{array}$ & $\begin{array}{c}M_{R}(\text { host }) \\
\text { (6) }\end{array}$ & $\begin{array}{c}M_{R}(\mathrm{QSO}) \\
(7)\end{array}$ & $\begin{array}{c}\text { Reference } \\
\text { (8) }\end{array}$ & $\begin{array}{c}L_{5 \mathrm{GHz}}^{\mathrm{ext}} \\
(9)\end{array}$ & $\begin{array}{c}\text { Reference } \\
(10)\end{array}$ \\
\hline 1 & $0133+207$ & $3 \mathrm{C} 47$ & $\mathrm{~S}$ & 0.425 & -23.57 & -24.27 & 2 & 26.71 & 6 \\
\hline 2 & $0137+012$ & PHL 1093 & $S$ & 0.258 & -23.74 & -23.67 & 2 & 27.07 & 7 \\
\hline 3 & $0137+3309$ & $3 \mathrm{C} 48$ & S & 0.367 & -25.03 & -25.92 & 2 & 27.52 & 6 \\
\hline 4 & $0202-765$ & PKS & S & 0.389 & -23.32 & -25.18 & 2 & $\ldots$ & \\
\hline 5 & $0312-770$ & PKS & $\mathrm{F}$ & 0.223 & -23.98 & -24.60 & 2 & $\ldots$ & \\
\hline 6 & $0340+048$ & $3 \mathrm{C} 93$ & $S$ & 0.357 & -23.93 & -23.71 & 2 & 25.56 & 8 \\
\hline 7 & $0405-123$ & OF-109 & $\mathrm{F}$ & 0.573 & -23.97 & -27.87 & 1 & 27.16 & 6 \\
\hline 8 & 0454-22 & PKS & S & 0.533 & -21.39 & -26.79 & 1 & 27.57 & 9 \\
\hline 9 & $0736+01$ & OI+061 & $\mathrm{F}$ & 0.191 & -23.78 & -24.23 & 2 & 24.32 & 6 \\
\hline 10 & $0837-120$ & $3 C 206$ & S & 0.198 & -23.29 & -24.23 & 2 & 26.03 & 6 \\
\hline 11 & $0903+169$ & $3 \mathrm{C} 215$ & S & 0.412 & -23.28 & -24.28 & 2 & 26.51 & 6 \\
\hline 12 & $1004+130$ & 4C 13.41 & S & 0.240 & -24.26 & -25.82 & 2 & 26.15 & 6 \\
\hline 13 & $1020-103$ & OL-133 & S & 0.197 & -23.36 & -23.47 & 3 & 24.83 & 13 \\
\hline 14 & $1138+0003$ & LBQS & $?$ & 0.500 & -23.97 & -24.35 & 4 & $\ldots$ & \\
\hline 15 & $1217+023$ & $\mathrm{ON}+029$ & $\mathrm{~F}$ & 0.239 & -23.71 & -24.40 & 3 & 25.45 & 6 \\
\hline 16 & $1218+1734$ & LBQS & $\mathrm{S}^{a}$ & 0.445 & -23.46 & -23.74 & 2 & 25.93 & 14 \\
\hline 17 & $1222+1235$ & LBQS & $\mathrm{S}^{b}$ & 0.412 & -24.02 & -24.24 & 2 & 25.96 & 14 \\
\hline 18 & $1226+023$ & $3 C 273$ & F & 0.158 & -24.44 & -27.39 & 2 & 27.04 & 6 \\
\hline 19 & $1230-0015$ & LBQS & S & 0.470 & -23.14 & -24.64 & 4 & $\ldots$ & \\
\hline 20 & $1250+568$ & 3C 277.1 & S & 0.321 & -22.96 & -23.29 & 2 & 26.73 & 6 \\
\hline 21 & $1302-102$ & OP-106 & S & 0.286 & -24.34 & -26.13 & 2 & 25.70 & 6 \\
\hline 22 & $1309+355$ & Ton 1565 & $\mathrm{~F}$ & 0.184 & -23.82 & -24.73 & 2 & 24.14 & 6 \\
\hline 23 & $1425+267$ & Ton 202 & S & 0.366 & -23.65 & -25.69 & 2 & 25.78 & 6 \\
\hline 24 & $1512+37$ & 4C 37.43 & S & 0.371 & -23.86 & -25.58 & 2 & 26.28 & 6 \\
\hline 25 & $1545+210$ & $3 \mathrm{C} 323.1$ & S & 0.266 & -23.53 & -25.14 & 2 & 26.42 & 6 \\
\hline 26 & $1548+114 \mathrm{~A}$ & $4 C+11.50$ & $\mathrm{~F}$ & 0.436 & -22.40 & -23.86 & 2 & 25.06 & 10 \\
\hline 27 & $1641+399$ & $3 \mathrm{C} 345$ & $\mathrm{~F}$ & 0.593 & -23.75 & -26.35 & 1 & 26.48 & 11 \\
\hline 28 & $1704+608$ & $3 C 351$ & $S$ & 0.371 & -24.79 & -26.16 & 2 & 26.76 & 6 \\
\hline 29 & $2128-123$ & OX -148 & $\mathrm{~F}$ & 0.501 & -22.82 & -27.11 & 5 & $\ldots$ & \\
\hline 30 & $2135-147$ & PHL 1657 & S & 0.200 & -23.43 & -24.16 & 2 & 26.34 & 6 \\
\hline 31 & $2141+175$ & OX 169 & $\mathrm{~F}$ & 0.213 & -23.38 & -24.79 & 2 & 26.33 & 6 \\
\hline 32 & $2201+315$ & 4C 31.63 & $\mathrm{~F}$ & 0.295 & -24.70 & -25.98 & 2 & 26.54 & 6 \\
\hline 33 & $2247+140$ & 4C 14.82 & $S$ & 0.237 & -23.52 & -24.10 & 2 & $\ldots$ & \\
\hline 34 & $2348+0210$ & LBQS & $?$ & 0.504 & -23.75 & -25.13 & 4 & $\ldots$ & \\
\hline 35 & 2349-014 & PB 5564 & S & 0.173 & -24.27 & -24.02 & 2 & $\ldots$ & \\
\hline 36 & $2351-0036$ & LBQS & $\mathrm{F}$ & 0.460 & -23.02 & -24.18 & 4 & $\ldots$ & \\
\hline 37 & $2355-082$ & PHL 6113 & $S$ & 0.210 & -23.62 & -23.06 & 3 & 28.01 & 12 \\
\hline
\end{tabular}

NoTES - Column (1) number; Col. (2): object; Col. (3): common name; Col. (4): type (based on the spectral index between 1.4 and 5 GHz; Col. (5): redshift; Col. (6) $R$-band absolute magnitude of the host galaxy; Col. (7): $R$-band absolute magnitude of the QSO; Col. (8): references for $M_{R}$; Col. (9): extended radio power (in units of $\mathrm{W} \mathrm{Hz}^{-1}$ ) at $5 \mathrm{GHz}$; Col. (10): references for $L_{5 \mathrm{GHz}}^{\text {ext }}$.

REFERENCES - (1) Boyce et al. (1999); (2) Hamilton et al. (2002); (3) Dunlop et al. (2003); (4) Hooper et al. (1997); (5) Boyce et al. (1998); (6) Xu et al. (1999); (7) Rector et al. (1995); (8) Bogers et al. (1994); (9) Aldcroft et al. (1993); (10) Hutchings et al. (1996); (11) White 1992); (12) Lister et al. (1994); (13) Brotherton (1996); (14) Hooper et al. (1996).

${ }^{a}$ Based on the index between 0.365 and $4.85 \mathrm{GHz}$ (Gregory \& Condon 1991; Douglas et al. 1996).

${ }^{b}$ Based on the index between 4.85 and $8.4 \mathrm{GHz}$ (Gregory \& Condon 1991 and Ref. 14).

There are two impediments in solving this problem. First, the mass of the central BH is highly uncertain. BH masses derived from fitting the "big blue bump" (Shields 1978; Malkan \& Sargent 1982), for example, are coupled to the accretion rate, which is not known independently. Second, the observed continuum of RLQs, even at optical and ultraviolet wavelengths, may be significantly contaminated by nonthermal emission from the relativistic jet. With the exception of objects with prominent big blue bumps, it is nontrivial to estimate the thermal component of the continuum. Thus, it is difficult to reliably deduce the state of the accretion disk, which can be described conveniently by the dimensionless accretion 


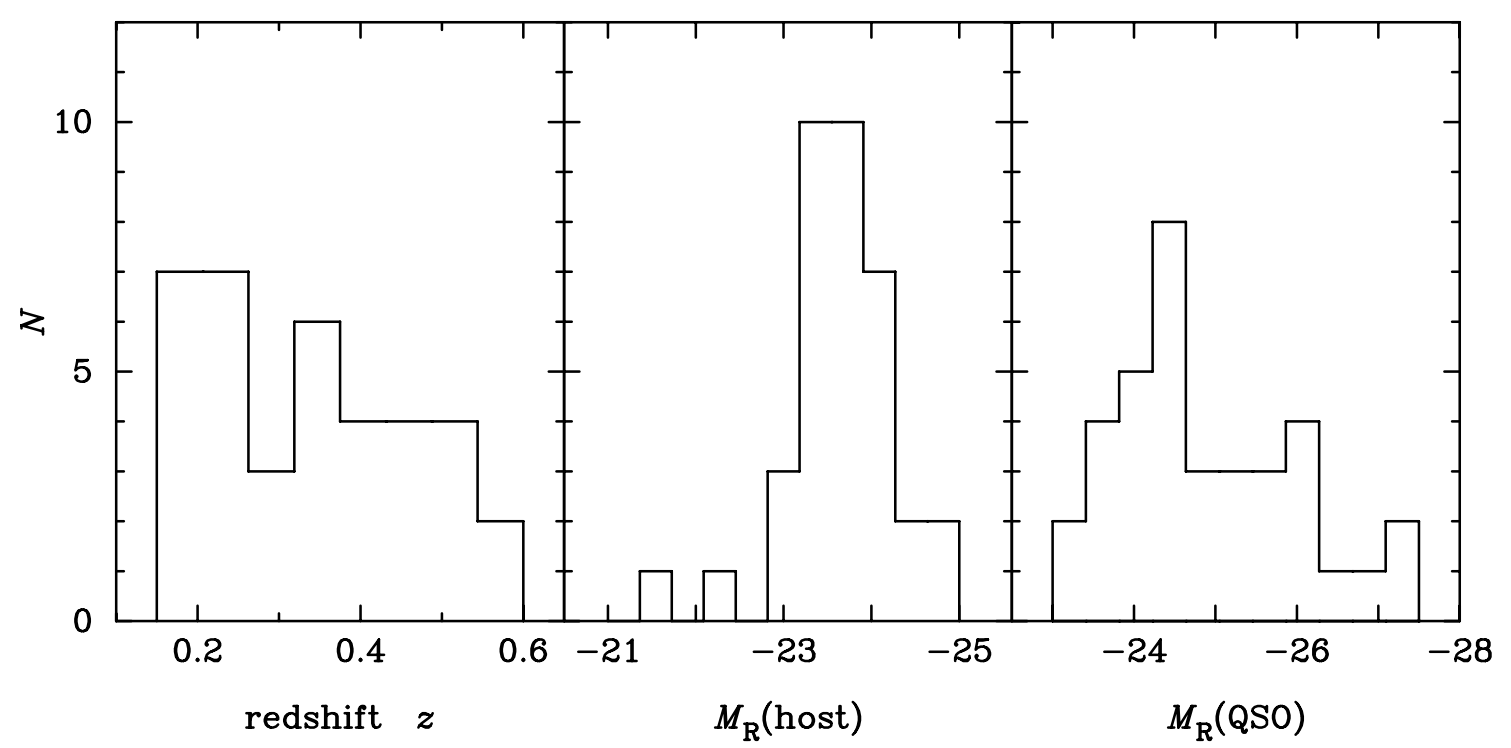

Fig. 1. Distribution of redshift, $M_{R}($ host $)$, and $M_{R}(\mathrm{QSO})$ for the present sample.

rate $\dot{m}=\dot{M} / \dot{M}_{\mathrm{Edd}}$, where $\dot{M}$ is the accretion rate, $\dot{M}_{\mathrm{Edd}}=$ $2.2 \times 10^{-8}(\eta / 0.1)^{-1}\left(M_{\mathrm{BH}} / M_{\odot}\right) M_{\odot} \mathrm{yr}^{-1}$ is the Eddington accretion rate defined by $\dot{M}_{\mathrm{Edd}}=L_{\mathrm{Edd}} / \eta c^{2}, L_{\mathrm{Edd}}=1.26 \times$ $10^{38}\left(M_{\mathrm{BH}} / M_{\odot}\right) \mathrm{erg} \mathrm{s}^{-1}$ is the Eddington luminosity, and $\eta$ is the radiative efficiency (Frank et al. 1992).

The situation has improved dramatically with the availability of Hubble Space Telescope (HST) observations of quasar host galaxies. Relatively reliable luminosities for the host galaxies are now available. From studies of nearby inactive galaxies, it has been established that the mass of the BH scales roughly linearly with the luminosity (or mass) of the bulge component of the host galaxy (Kormendy \& Richstone 1995; Magorrian et al. 1998; Ho 1999; Kormendy \& Gebhardt 2001). Thus, we can use the luminosities of the quasar host galaxies, which are largely dominated by the bulge component, to estimate $\mathrm{BH}$ masses for quasars.

Accretion disks have complicate structures for different $\dot{m}$ and the viscosity $\alpha$ (Chen et al. 1995). For a disk with a low accretion rate, three possible solutions have been suggested: optically thin advection-dominated accretion flows (ADAF; Narayan \& Yi 1994), advection-dominated inflow-outflows (ADIOs; Blandford \& Begelman 1999) and convection-dominated accretion flows (CDAF; Narayan et al. 2000; Quataert \& Gruzinov 2000). Whether low $\dot{m}$ necessarily leads to low $\eta$, however, remains controversial. When $\alpha^{2}<$ $\dot{m}<1$, the disk has a standard optically thick, geometrically thin structure (Shakura \& Sunyaev 1973, hereafter SS), which has been extensively studied. When $\dot{m}>1$, an optically thick ADAF or super-Eddington accretion flow (SEA) with slim geometry is formed (Begelman \& Meier 1982; Abramowicz et al. 1988; Chen \& Taam 1993). Such a disk has two possible structures: an inhomogeneous structure (split into rings) due to the photon-bubble instability (Begelman 2002), or a homogeneous disk with photon trapping (Wang \& Zhou 1999; Ohsuga et al. 2002). Once the mass of the BH and the accretion luminosity are known, we can constrain the state of the accretion disk, which provides possible clues to jet formation.
Recently, a number of authors have suggested that flatspectrum radio quasars, based on their very high kinetic luminosities, are powered by super-Eddington accretion flows (Ghisellini \& Celotti 2001; Cavaliere \& D'Elia 2002; Maraschi $\&$ Tavecchio 2003). It would be highly desirable to test this and other hypotheses by obtaining a reliable estimate of the accretion rates of RLQs. This is the purpose of this paper.

\section{The sample}

The most reliable measurement of quasar host galaxy luminosities currently come from HST observations. Our analysis uses RLQs with available HST imaging, based mainly on three samples (see Table 1): (1) Hamilton et al. (2002), (2) McLure et al. (2001) and Dunlop et al. (2003), and (3) Boyce et al. (1998, 1999). The $B$ and $V$ magnitudes are converted into the $R$ band following Fukugita et al. (1995). We adopt the results from Hamilton et al. (2002) if the objects have been measured several times in order to decrease the systematic errors. Unfortunately, systematic errors in host galaxy measurements are difficult to quantify, but uncertainties on the order of 0.1-0.3 mag are not unexpected. Table 1 also lists the absolute magnitudes of the quasar nuclei, based on spatial decomposition of the HST images. Finally, we give published values of the extended radio luminosity. Following standard practice, the objects are divided into two subclasses according to their radio spectral index $n$, defined by $F_{v} \propto v^{n}$ : flat-spectrum radio quasars (FSRQs) are those with $n \geq-0.5$, whereas steepspectrum radio quasars (SSRQs) have $n<-0.5$, where $n$ is generally calculated between 1.4 and $5 \mathrm{GHz}$. There are two objects without spectral index, but they do not change our conclusions (see Tables 2 and 3 ).

The redshift range of the sample is $0.16 \leq z \leq 0.57$, and the host galaxy absolute magnitudes span $-21.4 \geq M_{R} \geq-25.0$ (Fig. 1). For our present application, we use the empirical BH mass-bulge luminosity relation of McLure \& Dunlop (2002):

$\log \left(M_{\mathrm{BH}} / M_{\odot}\right)=-0.5 M_{R}-2.96$. 
Table 2. Line luminosities.

\begin{tabular}{|c|c|c|c|c|}
\hline $\begin{array}{l}\text { No. } \\
\text { (1) }\end{array}$ & $\begin{array}{c}\text { Lines } \\
(2)\end{array}$ & $\begin{array}{l}\text { Reference } \\
\text { (3) }\end{array}$ & $\begin{array}{c}\log \text { Flux }\left(\mathrm{erg} \mathrm{s}^{-1} \mathrm{~cm}^{-2}\right) \\
\text { (4) }\end{array}$ & $\begin{array}{c}\log \text { Luminosity }\left(\mathrm{erg} \mathrm{s}^{-1}\right) \\
\text { (5) }\end{array}$ \\
\hline 1 & $\mathrm{C}$ IV, Ly $\alpha$ & 15 & -11.74 & 45.22 \\
\hline 2 & $\mathrm{H} \alpha, \mathrm{H} \beta, \mathrm{H} \gamma$ & 1 & -11.42 & 45.09 \\
\hline 3 & $\mathrm{H} \gamma, \mathrm{C}$ IV, $\mathrm{Mg}$ II, Ly $\alpha$ & 16 & -11.49 & 45.34 \\
\hline 4 & $\mathrm{H} \beta, \mathrm{H} \gamma, \mathrm{Mg}$ II & 5 & -11.93 & 44.95 \\
\hline 5 & $\mathrm{C}$ IV, $\operatorname{Ly} \alpha$ & 7,11 & -11.41 & 44.96 \\
\hline 6 & $\mathrm{H} \alpha$ & 6 & -12.60 & 44.21 \\
\hline 7 & $\mathrm{H} \alpha, \mathrm{H} \beta, \mathrm{C}$ IV, Mg II, Ly $\alpha$ & $7,9,10,11,12$ & -11.21 & 46.03 \\
\hline 8 & $\mathrm{H} \beta, \mathrm{C}$ IV, Ly $\alpha$ & 9,11 & -11.64 & 45.53 \\
\hline 9 & $\mathrm{H} \alpha, \mathrm{H} \beta, \mathrm{H} \gamma, \mathrm{C}$ IV, Ly $\alpha$ & 8,11 & -11.80 & 44.43 \\
\hline 10 & $\mathrm{H} \alpha, \mathrm{H} \beta, \mathrm{H} \gamma, \mathrm{C}$ IV, Ly $\alpha$ & $8,10,11$ & -11.64 & 44.63 \\
\hline 11 & $\mathrm{H} \gamma, \mathrm{Mg}$ II & 16 & -11.95 & 44.98 \\
\hline 12 & $\mathrm{H} \alpha, \mathrm{H} \beta, \mathrm{C}$ IV, Mg II, Ly $\alpha$ & $8,10,11$ & -11.67 & 44.77 \\
\hline 13 & $\mathrm{H} \alpha, \mathrm{H} \beta, \mathrm{H} \gamma$ & 13 & -11.79 & 44.47 \\
\hline 15 & $\mathrm{H} \alpha, \mathrm{H} \beta, \mathrm{H} \gamma, \mathrm{C}$ IV, Ly $\alpha$ & $8,11,7$ & -11.87 & 44.57 \\
\hline 18 & $\mathrm{H} \beta, \mathrm{H} \gamma, \mathrm{C}$ IV, Mg II, Ly $\alpha$ & 3,14 & -10.28 & 45.79 \\
\hline 20 & $\mathrm{H} \alpha, \mathrm{H} \beta, \mathrm{H} \gamma, \mathrm{C}$ IV, Mg II & 8,15 & -11.96 & 44.75 \\
\hline 21 & $\mathrm{H} \beta, \mathrm{C}$ IV, Ly $\alpha$ & $7,9,11$ & -11.51 & 45.09 \\
\hline 22 & 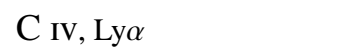 & 11 & -11.50 & 44.70 \\
\hline 23 & $\mathrm{H} \alpha, \mathrm{H} \beta, \mathrm{C}$ IV, $\operatorname{Mg}$ II, Ly $\alpha$ & 10,11 & -11.66 & 45.16 \\
\hline 24 & $\mathrm{H} \alpha, \mathrm{H} \beta, \mathrm{C}$ IV, Mg II, Ly $\alpha$ & $7,9,10,11$ & -11.59 & 45.25 \\
\hline 25 & $\mathrm{H} \beta, \mathrm{C}$ IV, Mg II, Ly $\alpha$ & $7,9,15$ & -11.11 & 45.42 \\
\hline 26 & $\mathrm{H} \beta, \mathrm{H} \gamma$ & 2 & -12.34 & 44.64 \\
\hline 27 & $\mathrm{H} \beta, \mathrm{H} \gamma, \mathrm{C}$ IV, $\mathrm{Ly} \alpha$ & 8,11 & -11.84 & 45.44 \\
\hline 28 & $\mathrm{H} \alpha, \mathrm{H} \beta, \mathrm{C}$ IV, Mg II, Ly $\alpha$ & $7,9,10,11$ & -11.63 & 45.21 \\
\hline 29 & $\mathrm{H} \beta, \mathrm{H} \gamma, \mathrm{C}$ IV, Mg II, Ly $\alpha$ & $2,7,11,12$ & -12.04 & 45.08 \\
\hline 30 & $\mathrm{H} \beta, \mathrm{C}$ IV, Ly $\alpha$ & $7,9,11$ & -11.40 & 44.87 \\
\hline 31 & $\mathrm{H} \beta, \mathrm{C}$ IV, Ly $\alpha$ & $7,9,11$ & -11.69 & 44.64 \\
\hline 32 & $\mathrm{H} \alpha, \mathrm{H} \beta, \mathrm{H} \gamma, \mathrm{C}$ IV, $\mathrm{Ly} \alpha$ & $7,8,9,10,11$ & -11.31 & 45.31 \\
\hline 33 & $\mathrm{H} \beta$ & 4 & -12.22 & 44.21 \\
\hline 35 & $\mathrm{H} \beta$ & 4 & -11.25 & 44.89 \\
\hline
\end{tabular}

Notes - Colomn (1) number; Col. (2) emission lines; Col. (3) references; Col. (4) the derived total line fluxes; and Col. (5) the derived total line luminosity.

REFERENCES - (1) Baldwin (1975); (2) Bergeron \& Kunth (1984); (3) Corbin (1992); (4) Corbin (1997); (5) Danziger \& Goss (1983); (6) Eracleous \& Halpern (1994); (7) Gondhalekar (1990); (8) Jackson \& Browne (1991); (9) Marziani et al. (1996); (10) Neugebauer et al. (1979); (11) Osmer et al. (1994); (12) Oke et al. (1984); (13) Scarpa \& Falomo (1997); (14) Stockton \& MacKenty (1987); (15) Wills et al. (1995); (16) Wilkes et al. (1999).

For ease of comparison with literature results, distancedependent quantities assume a Hubble constant of $H_{0}=$ $50 \mathrm{~km} \mathrm{~s}^{-1} \mathrm{Mpc}^{-1}$ and a deceleration parameter of $q_{0}=0.5$.

\section{States of the accretion disk}

The observed optical-UV continuum of RLQs may be contaminated by nonthermal emission from the relativistic jet, rendering it difficult to estimate the thermal contribution to the continuum, and hence to estimate accretion rates. On the other hand, quasars, independent of their radio strength, usually have strong, well-observed broad emission lines, which leads to the possibility of using the broad-line region (BLR) emission-line strength to indirectly estimate the ionizing luminosity. Following Celotti et al. (1997), this is the approach we will take here. Celotti et al. (1997) showed that the BLR luminosities in RLQs are roughly equal to the kinetic luminosities of their jets. We assume that the BLR clouds are most likely photoionized by the thermal emission from the accretion disk, rather than by nonthermal emission from the jet. This 
Table 3. Results.

\begin{tabular}{|c|c|c|c|c|}
\hline Object & $\begin{array}{c}\log M_{\mathrm{BH}} \\
\left(M_{\odot}\right)\end{array}$ & $\begin{array}{l}\log \Lambda \\
\left(\mathrm{g} \mathrm{s}^{-1}\right)\end{array}$ & $\log \lambda$ & 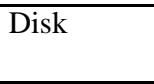 \\
\hline 1 & 8.82 & 24.27 & -1.71 & SS \\
\hline 2 & 8.91 & 24.14 & -1.92 & SS \\
\hline 3 & 9.56 & 24.39 & -2.32 & $\mathrm{SS}+\mathrm{ADAF}$ \\
\hline 4 & 8.70 & 24.00 & -1.85 & SS \\
\hline 5 & 9.03 & 24.01 & -2.17 & $\mathrm{SS}+\mathrm{ADAF}$ \\
\hline 6 & 9.01 & 23.26 & -2.90 & ADAF \\
\hline 7 & 9.02 & 25.08 & -1.10 & SS \\
\hline 8 & 7.73 & 24.58 & -0.31 & SEA \\
\hline 9 & 8.93 & 23.48 & -2.60 & $\mathrm{SS}+\mathrm{ADAF}$ \\
\hline 10 & 8.69 & 23.68 & -2.16 & $\mathrm{SS}+\mathrm{ADAF}$ \\
\hline 11 & 8.68 & 24.03 & -1.80 & SS \\
\hline 12 & 9.17 & 23.82 & -2.50 & $\mathrm{SS}+\mathrm{ADAF}$ \\
\hline 13 & 8.72 & 23.52 & -2.35 & $\mathrm{SS}+\mathrm{ADAF}$ \\
\hline 15 & 8.89 & 23.62 & -2.43 & $\mathrm{SS}+\mathrm{ADAF}$ \\
\hline 18 & 9.26 & 24.84 & -1.57 & SS \\
\hline 20 & 8.52 & 23.80 & -1.87 & SS \\
\hline 21 & 9.21 & 24.14 & -2.22 & $\mathrm{SS}+\mathrm{ADAF}$ \\
\hline 22 & 8.95 & 23.75 & -2.35 & $\mathrm{SS}+\mathrm{ADAF}$ \\
\hline 23 & 8.86 & 24.21 & -1.81 & SS \\
\hline 24 & 8.97 & 24.30 & -1.82 & SS \\
\hline 25 & 8.81 & 24.47 & -1.49 & SS \\
\hline 26 & 8.24 & 23.69 & -1.70 & SS \\
\hline 27 & 8.91 & 24.49 & -1.58 & SS \\
\hline 28 & 9.44 & 24.26 & -2.33 & $\mathrm{SS}+\mathrm{ADAF}$ \\
\hline 29 & 8.45 & 24.13 & -1.47 & SS \\
\hline 30 & 8.76 & 23.92 & -1.99 & SS \\
\hline 31 & 8.73 & 23.69 & -2.19 & $\mathrm{SS}+\mathrm{ADAF}$ \\
\hline 32 & 9.39 & 24.36 & -2.18 & $\mathrm{SS}+\mathrm{ADAF}$ \\
\hline 33 & 8.80 & 23.26 & -2.69 & $\mathrm{SS}+\mathrm{ADAF}$ \\
\hline 35 & 9.18 & 23.94 & -2.39 & $\mathrm{SS}+\mathrm{ADAF}$ \\
\hline
\end{tabular}

assumption is supported by that the beamed radiation from the jet has great difficulties to ionize the clouds distributed within a large solid angle to produce a reasonable range of ionization parameter for emission lines. There is also ample evidence that BLR emission lines are photoionized by a thermal continuum (see, e.g., Netzer 1990). The underlying assumption here should be quite safe in statistical test.

Table 2 lists emission-line fluxes we were able to assemble for our sample. We choose prominent broad emission lines, such as $\mathrm{H} \alpha, \mathrm{H} \beta, \mathrm{H} \gamma, \mathrm{C}$ IV, Mg II and $\mathrm{Ly} \alpha$, that account for a large fraction of the total line luminosity. The overall similarity of the emission-line spectra of quasars suggests that the underlying physical conditions of their BLR are quite similar (Netzer 1990; Francis et al. 1991; Boroson \& Green 1992; Zheng et al. 1997). To first order, therefore, the composite spectrum of quasars (Francis et al. 1991) may be used as a reasonable template to calculate the total BLR luminosity (Celotti et al. 1997). Then, from the relative contribution of individual lines to the total BLR luminosity, we obtain the total line luminosity $L_{\text {lines }}$ (Table 2 ).

The fraction $\xi$ of the thermal disk emission reprocessed by the BLR clouds is roughly equal to the cloud covering factor. This assumption should be statistically valid for large samples since in steady state the energy absorbed by the BLR clouds is approximately that radiated as line emission. Thus, the thermal emission from the accretion disk is

$L_{\text {disk }}=\xi^{-1} L_{\text {lines }}$.

According to Netzer (1990), $\xi \approx 0.1$.

We recognize that, for any individual object, variability and internal reddening may introduce uncertainties into $L_{\text {lines }}$. Source-to-source variations in the covering factor or reprocessing efficiency may also add additional scatter. It is hoped, however, that the overall statistics of a large sample will not be severely affected and that they are at least internally consistent.

Following Wang et al. (2002), we define the line accretion rate and its dimensionless form as

$\Lambda=\frac{L_{\text {lines }}}{c^{2}} ; \quad \lambda=\frac{L_{\text {lines }}}{L_{\text {Edd }}}$.

The relation between $\lambda$ and the dimensionless accretion rate $\dot{m}$, for an optically thin ADAF, is given by (Wang et al. 2002)

$\dot{m}=2.17 \times 10^{-2} \alpha_{0.3} \xi_{-1}^{-1 / 2} \lambda_{-4}^{1 / 2}$.

This expression assumes that the total luminosity from the ADAF is $L_{\text {disk }} \propto \alpha^{-2} M_{\mathrm{BH}} \dot{m}^{2}$ (Mahadevan 1997), $\alpha_{0.3}=\alpha / 0.3$, $\xi_{-1}=\xi / 0.1$, and $\lambda_{-4}=\lambda / 10^{-4}$. A necessary condition for the presence of an optically thin ADAF is $\dot{m} \leq \alpha^{2}$ (Narayan et al. 1998). Equation (4) can then be rewritten as

$\lambda_{1}=1.72 \times 10^{-3} \xi_{-1} \alpha_{0.3}^{2}$.

Optically thin ADAFs require $\lambda<\lambda_{1}$.

Accretion disks in the optically thick, geometrically thin SS regime obey

$\dot{m}=\frac{L_{\text {line }}}{\xi L_{\text {Edd }}}=10 \xi_{-1}^{-1} \lambda$,

and the condition $1>\dot{m} \geq \alpha^{2}$ gives

$\lambda_{2}=9.0 \times 10^{-3} \xi_{-1} \alpha_{0.3}^{2}$,

above which a standard disk can exist. It is interesting to note that in the transition region between $\lambda_{1}$ and $\lambda_{2}$ the accretion flow may be in a hybrid state in which a standard disk coexists with an ADAF. The possibility of hybrid states in AGN accretion disks has been discussed by, among others, Quataert et al. (1999), Rózanska \& Czerny (2000), Ho et al. (2000), and Ho (2002a). A transition from an SS disk to an ADAF is possible (Gu \& Lu 2000), perhaps via evaporation (Liu et al. 1999). The transition radius depends on the accretion rate, the $\mathrm{BH}$ mass, and viscosity. The disk structure, however, is complicated in such a regime, largely due to uncertainties in the viscosity.

When the accretion rate reaches $\dot{m} \geq 1$, we have

$\lambda_{3}=0.1 \xi_{-1}$,

in which case we use

$E_{\mathrm{R}}=\frac{L_{\text {line }}}{\xi L_{\mathrm{Edd}}}$

to describe the accretion state, since $\dot{m}>E_{R}$ in the superEddington regime in which photon trapping efficiently lowers the radiated luminosity (Wang \& Zhou 1999; Ohsuga et al. 2002). 

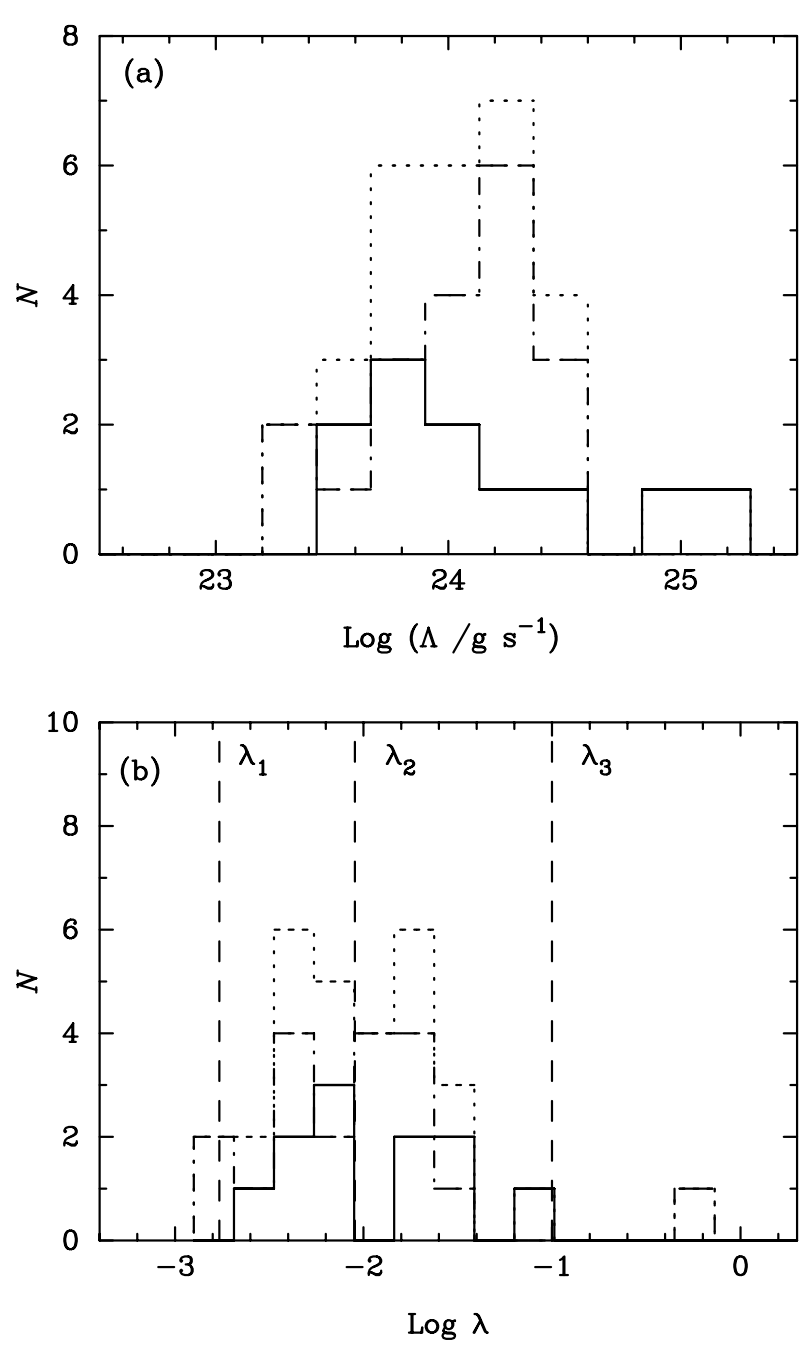

Fig. 2. The distribution of a) $\Lambda$ and b) $\lambda$. The solid histograms plot the FSRQs, and the dotted-dashed histograms show the SSRQs. The dotted histograms show the total distribution for all RLQs (FSRQs and SSRQs). The distribution of $\lambda$ is divided into four regions, corresponding to different states of the accretion disks: (1) $\lambda<\lambda_{1}$ (pure ADAF); (2) $\lambda_{1} \leq \lambda \leq \lambda_{2}$ (ADAF+SS); (3) $\lambda_{2} \leq \lambda \leq \lambda_{3}$ (SS); (4) $\lambda \geq \lambda_{3}$ (SEA).

Our estimation of the parameter $\lambda$ are subject to at least three sources of uncertainty. First, the total line luminosity is estimated from only a limited number of emission lines. Since the relative strengths of the main strong lines are relatively well determined, and quasars generally have fairly uniform spectra (e.g., Francis et al. 1991; Zheng et al. 1997), this should not introduce a large source of uncertainty. More worrisome is our assumption of a constant cloud covering factor (10\%), which in reality is likely to have an intrinsic dispersion. And third, even if we assume that the host galaxy magnitudes are perfectly measured, the $M_{R}-M_{\mathrm{BH}}$ relation still has a scatter of $\sim 0.4$ dex (McLure \& Dunlop 2002). For concreteness, we assume that any individual value of $\lambda$ may have an uncertainty of $\sim 0.5 \mathrm{dex}$. Although this is not negligible, fortunately it is not sufficiently large to obscure gross statistical trends in our sample. However, we caution against a literal interpretation of the individual values of $\lambda$ and $\Lambda$ listed in Table 3 .
The three critical values of $\lambda$ define four regimes in accretion states, as shown in Fig. 2b. The average values of $\Lambda$ for FSRQs and SSRQs are, respectively, $\langle\log \Lambda\rangle_{\mathrm{FSRQ}}=24.0$ and $\langle\log \Lambda\rangle_{\text {SSRQ }}=24.1$. The corresponding values for $\lambda$ are $\langle\log \lambda\rangle_{\mathrm{FSRQ}}=-2.20$ and $\langle\log \lambda\rangle_{\mathrm{SSRQ}}=-2.05$. Given the uncertainties just discussed, the two subsamples appear to be statistically indistinguishable.

Wang et al. (2002) present the distributions of $\Lambda$ and $\lambda$ for BL Lac objects and conclude that all BL Lacs have ADAFs. Comparing those objects with the RLQs presented here, we find that the distributions of $\Lambda$ overlap significantly, increasing smoothly from BL Lacs to RLQs. This was noted by Scarpa \& Falomo (1997), who used only Mg II as the luminosity indicator. Our result considerably strengthens theirs. A similar behavior is seen in the distribution of $\lambda$. As we discuss later, these trends suggest that an evolutionary sequence, characterized by different accretion states, may connect BL Lac objects to RLQs.

The normalized mass accretion rates, $\dot{m}=\xi^{-1} \lambda \approx 10 \lambda$, span over a relatively large range, from $10^{-2}$ to $10^{0.7}$. Some of the FSRQs in our sample fall in the range of objects studied by Cavaliere \& D'Elia (2002), but we suggest that some of FSRQs, like SSRQs, may have hybrid ADAF+SS disks. With the similarity in $\dot{m}$ between FSRQs and SSRQs, the only difference between FSRQs and SSRQs is geometrical orientation, which has been suggested (Urry \& Padovani 1995).

Cavaliere \& D'Elia (200202) suggest that an evolutionary sequence may be defined for blazars, depending on their dimensionless accretion rates. FSRQs have $\dot{m} \approx 10^{-1}$, whereas BL Lac objects have $\dot{m} \approx 10^{-3}$, the two populations separated by a significant gap. By contrast, our analysis suggests that BL Lacs and RLQs define a continuous sequence in accretion rate. Although our conclusion is based on two heterogeneous samples, we note that it is in general agreement with the evolutionary scenario proposed by Böttcher \& Dermer (2002).

The states of the accretion disk listed in Table 3 can be tested by $\mathrm{Fe} \mathrm{K} \alpha$ line observations. Differences in the X-ray properties between radio-loud and radio-quite quasars have been discussed by Sambruna et al. (1999) and Reeves \& Turner (2000). The most important findings in these studies is that the Fe K $\alpha$ line tends to have complex profiles. Only a few RLQs have broad $\mathrm{Fe} \mathrm{K} \alpha$ profiles, most being undetected (Sambruna et al. 1999; Reeves \& Turner 2000). There is also a clear trend that more radio-loud objects tend to have lower $\mathrm{Fe} \mathrm{K} \alpha$ equivalent widths. By analogy with the interpretation for the relativistically broadened Fe lines in Seyfert nuclei (e.g., Nandra et al. 1997), RLQs with strong broad $\mathrm{Fe} \mathrm{K} \alpha$ lines may be in the SS state, while those lacking such a feature may be in a pure $\mathrm{ADAF}$ or in an ADAF+SS state, according to the classification suggested in this study. This interpretation, although appealing, may not be unique. Recall that among Seyfert galaxies and radio-quiet quasars there exists an inverse correlation between Fe line strength and X-ray luminosity (Iwasawa \& Taniguchi 1993), an effect that can be attributed to luminosity-dependent changes in the ionization state of the surface of the disk (Reeves $\&$ Turner 2000). Alternatively, perhaps strong relativistic jets physically modify the structure of the innermost part of the 


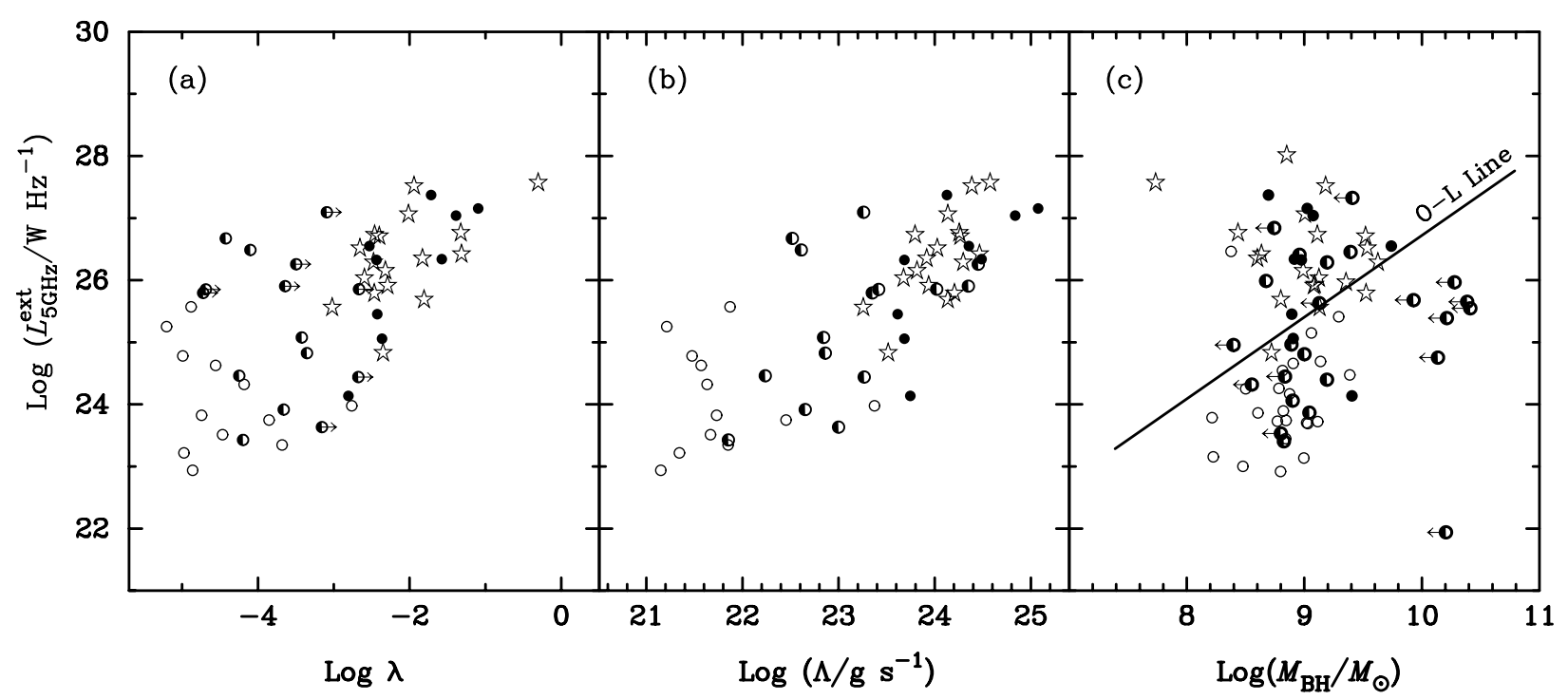

Fig. 3. The distribution of extended radio power at $5 \mathrm{GHz}$ versus the a) $\lambda$, b) $\Lambda$, and c) $M_{\mathrm{BH}}$. The filled circles show the FSRQs, and SSRQs are denoted as open stars. The open and semi-filled circles represent high frequency-peaked and low frequency-peaked BL Lacs, respectively; the radio data for these objects are taken from Urry et al. (2000), and values for $\lambda, \Lambda$, and $M_{\mathrm{BH}}$ are taken from Wang et al. (2002). The diagonal line in panel c) is the empirical division between FR I and FR II radio galaxies in the diagram of extended radio power at $1.4 \mathrm{GHz}$ versus optical $R$-band absolute magnitude, as presented by Ledlow \& Owen (1996); see text for details.

disk, thereby altering or suppressing the iron-line emitting region (see Sect. 4.1.3).

We note that values of $\lambda_{1}$ and $\lambda_{2}$ are sensitive to $\alpha$. The most likely value of $\alpha$ is 0.3 (Narayan et al. 1998). However if $\alpha=0.1$, it will follow that $\lambda_{1}=1.91 \times 10^{-4} \xi_{-1} \alpha_{0.1}^{2}$ and $\lambda_{2}=1.0 \times 10^{-3} \xi_{-1} \alpha_{0.1}^{2}$. All the disks listed as hybrid models of SS and ADAF will be SS disk in Table 3. In any case, this does not affect the conclusion that most of the objects have the standard accretion disks.

There is only one possible super-Eddington accretor (PKS 0454-22) in the present sample. This nicely agrees with the result from an independent test by Wang (2003), in which super-Eddington accretors have been searched in the present sample and no super-Eddington accretor has been found. He used the theoretical spectra emergent from slim disks (Wang et al. 1999) and the empirical reverberation relation to find a limit relation bewteen the $\mathrm{BH}$ mass and $\mathrm{H} \beta$ width as $M_{\mathrm{BH}}=(2.9-12.6) \times 10^{6} M_{\odot}\left(v_{\mathrm{FWHM}} / 10^{3} \mathrm{~km} \mathrm{~s}^{-1}\right)^{6.67}$ in AGNs with super-Eddington rates. With the help of Eq. (1), the BH masses can be obtained, he found all the objects in the present sample are located below this limit.

It is worth noting that, with the exception of 3 C 273 , our sample lacks very powerful FSRQs. This is an observational selection effect. It is difficult to measure the host galaxies of the most powerful quasars because of their very bright nuclei. We will later return to the issue of whether there exists superEddington RLQs that appear as very powerful FSRQs.

\section{Discussion}

\subsection{Jet formation from the disk}

Two processes have been widely advocated for jet formation in AGNs: (1) energy extraction from the spin of the $\mathrm{BH}$
(Blandford \& Znajek 1977, hereafter BZ) and (2) energy extraction from a disk wind (Blandford \& Payne 1982, hereafter BP). Several variants of the BZ and BP models have been proposed. The results of the present study allow us to set some constraints on the theoretical models. In their investigation of the BZ process in the regime of the SS disk, Livio et al. (1999) conclude that both the BZ and BP power, even for Kerr BHs, are negligible compared with the radiative output from the accretion disk itself. The reason is that geometrically thin disks cannot produce a strong enough poloidal magnetic field for the energy extraction to be effective.

\subsubsection{Model of Ghosh \& Abramowicz}

Ghosh \& Abramowicz (1997) assume that the poloidal magnetic field can be parameterized as $B_{\mathrm{p}}^{2} \propto \alpha P_{\max }$, where $P_{\max }$ is the maximum pressure in the inner region if the accretion rate $\dot{m}>10^{-3}\left(\alpha_{-2} m_{9}\right)^{-1 / 8}$, with $\alpha_{-2}=\alpha / 10^{-2}$ and $m_{9}=$ $M_{\mathrm{BH}} / 10^{9} M_{\odot}$. In such a region, the radiation pressure dominates over the gas pressure and is independent of the accretion rate. Then, the BZ power is given by

$L_{\mathrm{BZ}}=2 \times 10^{45} \mathrm{~m}_{9} \mathrm{j}^{2} \mathrm{erg} \mathrm{s}^{-1}$,

where $j=J_{\mathrm{BH}} /\left(G M_{\mathrm{BH}}^{2} c^{-1}\right)$ is the specific angular momentum. We can see that the BZ power is independent of the accretion rate, but very sensitive to the spin. The extended radio power is expected to be proportional to $L_{\mathrm{BZ}}$ (Meier 1999). For our sample, Fig. 3 shows that the extended radio powers span $\sim 5$ orders of magnitude, whereas the BH masses are confined to within roughly a factor of 10 . This means that the BHs should have spins in the range $10^{-2}<j<1.0$. Such a large range of values for the spin, however, is not expected in RLQs (Wilson \& Colbert 1995). The model of Ghosh \& Abramowicz (1997), therefore, seems to be disfavored by the present data. 
Additionally, the results of Lacy et al. (2001) from FIRST Bright Quasar Survey show that the radio emission depends on the accretion rate $\left(\propto \dot{m}^{1.0}\right)$, which does not support Eq. (10). As pointed out by Livio et al. (1999), Eq. (10) overestimates the BZ power because the poloidal magnetic field should be $B_{\mathrm{p}} \approx(H / R) B_{\phi}$ rather than simply $B_{\mathrm{p}}^{2} / 8 \pi \approx P_{\max }$.

\subsubsection{Model of Meier}

Meier (2001) considers energy extraction from the spin of the BH and from the disk itself, for both an SS disk and an ADAF. His model uses the middle-region solution for the SS disk and a self-similar solution for the ADAF; it considers both Schwarzschild and Kerr BHs. In this model the jet power for a Schwarzschild BH is only $L_{\text {jet }}=10^{41.7} \mathrm{erg} \mathrm{s}^{-1}$ if $M_{\mathrm{BH}}=10^{9} M_{\odot}$ and $\dot{m}=0.1$, whereas $L_{\mathrm{jet}}=10^{42.7} \mathrm{erg} \mathrm{s}^{-1}$ for a Kerr BH with the same mass and accretion rate. The present data clearly do not support this model because many RLQs have SS disks that support much higher luminosities. When the accretion disk is in an ADAF state, for example, an accretion rate of $\dot{m}=10^{-2.5}$ onto a Schwarzschild BH radiates $\sim 10^{42.3} \mathrm{erg} \mathrm{s}^{-1}$, which may generate a radio power of $10^{24.3} \epsilon_{-2} \mathrm{~W} \mathrm{~Hz}^{-1}$ at $1 \mathrm{GHz}$ in the extended lobes, where $\epsilon_{-2}=\epsilon / 10^{-2}$ is the fraction of the total jet power radiated in the radio band (Meier 1999). This can account for the radio emission from BL Lac objects shown in Fig. 3; it also implies that the BHs of BL Lac objects may not necessarily have large spins. The jet power from an ADAF surrounding a Kerr BH is approximately $10^{44.1} \mathrm{erg} \mathrm{s}^{-1}$, corresponding to a radio power of $10^{26.1} \epsilon_{-2} \mathrm{~W} \mathrm{~Hz}^{-1}$ at $1 \mathrm{GHz}$. This can account for RLQs in the $\mathrm{ADAF}+\mathrm{SS}$ regime. But how do we explain those RLQs that have much higher radio powers and that contain pure SS disks?

\subsubsection{An improved model}

From the above discussion, it is evident that the power output of the jet, whether generated through the BZ or BP processes, depends both on the model adopted for the accretion disk and the parameterization used for the poloidal magnetic field. Below we derive an improved formula for the jet power.

Following Meier (2001), the magnetohydrodynamic (MHD) power of the jet is given by

$L_{\mathrm{jet}}=\frac{1}{32 c} B_{\mathrm{p}}^{2} R_{0}^{4} \Omega_{0}^{2}$,

where $B_{\mathrm{p}}$ is the poloidal magnetic field and $\Omega_{0}$ is the Keplerian angular velocity at the radius $R_{0}$, where the jet develops. Both the $\mathrm{BZ}$ and the $\mathrm{BP}$ process need a strong poloidal magnetic field $B_{\mathrm{p}}$, which is produced by the toroidal magnetic field $B_{\phi}$. Livio et al. (1999) showed that

$B_{\mathrm{p}} \approx\left(\frac{H}{R}\right)^{n} B_{\phi}$,

where $H$ is the height of the disk at radius $R$ and the index $n \approx 1$. Since $H / R \ll 1$ for an SS disk, it is generally believed that $B_{\mathrm{p}} \ll B_{\phi}$, and hence that the extracted energy must be small (Livio et al. 1999; Meier 1999, 2001). However we should note that $H / R \approx 0.01$ is only valid in the middle and outer regions of the disk; in the inner region, $H / R \approx 1$. Meier (2001) argues that this region is thermally unstable. He only uses the middle-region solution in his calculations, and consequently obtains a reduced power from the BZ process.

The thermal instability arises from insufficient cooling. It is well known that such an instability in the innermost regions of the disk can be suppressed by advection, which acts as an efficient cooling process (Abramowicz et al. 1988; Chen \& Taam 1993). Advection mainly converts the heat of the accreting gas into entropy, rather than radiation. Consequently, the sound speed $\left(c_{\mathrm{s}}\right)$ and the scale height $H$ are increased. It would be of interest to include the role of advection cooling in the calculation of the $\mathrm{BZ}$ power.

We follow Chen's (1995) treatment of advection cooling in determining the structure of the disk. The disk is assumed to undergo Keplerian rotation at an angular velocity $\Omega_{\mathrm{K}}$, and we assume conservation of mass, conservation of angular momentum, and vertical equilibrium. Advection is included as local cooling, and we neglect the corona. The energy balance between the heat production $\left(Q_{\text {vis }}\right)$ and cooling can be written as

$Q_{\text {vis }}=Q_{\text {rad }}+Q_{\text {adv }}$,

where $Q_{\mathrm{rad}}$ and $Q_{\mathrm{adv}}$ represent radiative and advection cooling, respectively. The viscous heating rate per unit area is $Q_{\text {vis }}=3 \dot{M} \Omega^{2} f g / 4 \pi$, where the correction terms $f \approx 1$ and $g \approx 1$ are due to the inner boundary condition and the pseudoNewtonian potential (Chen 1995). Advection cooling can be expressed as $Q_{\mathrm{adv}}=\dot{M} c_{\mathrm{s}}^{2} \zeta / 2 \pi R^{2}, \zeta=5.5$ being the advection factor. With the diffusion approximation and the assumption of radiation pressure dominance, we have $Q_{\mathrm{rad}}=$ $4 a c T^{4} / 3 \kappa_{\mathrm{es}} \Sigma=2 c \Omega_{\mathrm{K}} c_{\mathrm{s}} / \kappa_{\mathrm{es}}$, where $\Sigma$ is the surface density of the disk, $a=7.56 \times 10^{-15}$ is the radiation density constant and $\kappa_{\mathrm{es}}=0.34$ is the electron-scattering opacity. With the help of the equations for mass and angular momentum conservation and vertical equilibrium, Eq. (13) reduces to

$\frac{\zeta \dot{M}}{2 \pi R^{2}} c_{\mathrm{s}}^{2}+\frac{2 c \Omega_{\mathrm{K}}}{\kappa_{\mathrm{es}}} c_{\mathrm{s}}-\frac{3 \dot{M}}{4 \pi} \Omega_{\mathrm{K}}^{2}=0$.

The first term represents advection cooling, which is only important at small radii or for high accretion rates, and the second represents radiative cooling. Neglecting the first term yields the standard solution for an SS disk, whereas neglecting the second gives the solution for an optically thick ADAF (or SEA). Advection cooling helps to suppress thermal instabilities that develop in the inner regions of the disk.

The solutions of the disk give the sound speed

$a_{\mathrm{s}}=0.09 \dot{m}_{-1}^{-1} r^{0.5}\left\{\sqrt{1+33 \dot{m}_{-1}^{2} r^{-2}}-1\right\}$,

where $a_{\mathrm{s}}=c_{\mathrm{s}} / c$ and $\dot{m}_{-1}=\dot{m} / 0.1$, the pressure

$P=1.76 \times 10^{7} \alpha^{-1} \dot{m}_{-1} m_{9}^{-1} a_{\mathrm{s}}^{-1} r^{-3}$ dyne $\mathrm{cm}^{-2}$,

and the vertical half-thickness

$\frac{H}{R}=a_{\mathrm{s}} r^{1 / 2}$,

where $r=R / R_{g}$ and $R_{g}=G M_{\mathrm{BH}} / c^{2}$. With the help of $B_{\phi}^{2} / 8 \pi \approx \alpha P$ and Eq. (12), we have

$B_{\mathrm{p}}=2.02 \times 10^{4} \dot{m}_{-1}^{1 / 2} m_{9}^{-1 / 2} a_{\mathrm{s}}^{1 / 2} r^{-1}$ Gauss. 
Following Meier (2001), we take the characteristic radius $R_{0}=7 R_{g}$ and $R_{0}=1.5 R_{g}$ for a Schwarzschild and Kerr $\mathrm{BH}$, respectively, and the angular velocity of the metric in the Boyer-Lindquist frame $\omega=-g_{\phi t} / g_{\phi \phi} \approx 0.3 j\left(G M_{\mathrm{BH}} / c^{3}\right)^{-1}$. We finally get the jet power from the accretion system. For a Schwarzschild BH,

$L_{\text {jet }}=8.60 \times 10^{43} m_{9} \dot{m}_{-1} \mathrm{erg} \mathrm{s}^{-1}$,

and for a Kerr BH,

$L_{\text {jet }}=1.87 \times 10^{45} m_{9} \dot{m}_{-1}\left(1+1.1 j+0.3 j^{2}\right) \mathrm{erg} \mathrm{s}^{-1}$.

Considering that the bolometric luminosity from accretion is $1.38 \times 10^{45} m_{9} \dot{m}_{-1} \mathrm{erg} \mathrm{s}^{-1}$, the ratio of the jet power to accretion power for a Schwarzschild BH is roughly $4 \times 10^{-2}$, whereas for a Kerr BH it is $\sim 1.0$.

The output powers given by Eqs. (19) and (20) are different from those found by Ghosh \& Abramowicz (1997) and Meier (2001). First, the energy output based on Eqs. (19) and (20) depends on the accretion rate, whereas the model of Ghosh \& Abramowicz (1997) does not. Second, the output is much higher than that given by Meier (2001) for the standard accretion disk model.

The MHD power for a Schwarzschild $\mathrm{BH}$ is due to the $\mathrm{BP}$ process. For a Kerr $\mathrm{BH}$, the $\mathrm{BP}$ process also depends strongly on the spin because the metric itself drags the MHD fluid, which then contributes to the rotation of the magnetic field; thus, Eq. (20) covers both the BP and BZ processes. The above levels of MHD power, which is extracted from the BH and the disk in the standard SS regime, are sufficient to account for the highest radio luminosities seen in RLQs.

As shown by $\mathrm{Xu}$ et al. (1999), the mass outflow rate from the jet is given by

$\dot{M}_{\text {jet }} \approx\left(\frac{H}{R}\right)^{2 \delta-3} \dot{M}$

where $\delta$ is a constant from 1.7-3.4. For Kerr BHs, the characteristic radius $r_{0}=1.5$ leads to $a_{\mathrm{s}} \approx 0.65 r^{-0.5}$, and hence $H / R \approx 0.65$ in the jet-forming region. We then have $\dot{M}_{\text {jet }} \approx$ $(0.19-0.84) \dot{M}$ : the mass outflow rate of the jet is a large fraction of the accretion rate. If the jet-formation region is an ADAF, the outflow rate will be even higher than this because $H / R \approx 1$. We note that such a large outflow rate may have implications for observations of the broad $\mathrm{Fe} \mathrm{K} \alpha$ line in radioloud AGNs. The region of the disk that emits the red wing of the broad line roughly coincides with the region where the relativistic jet is formed. If strong winds accompany jets, they can substantially alter the inner structure of the disk. This may be at least partly responsible for the observed diversity of Fe-line strengths and the complexity of their profiles in RLQs (Reeves $\&$ Turner 2000).

We note that Eq. (21) is not self-consistently obtained from the disk model. Such a high outflow rate may take place in ADIOS models. Here we stress that the Fe $\mathrm{K} \alpha$ line in RLQs may be strongly affected by the outflow. The case of 3C 120 adds credibility to the picture that jet formation influences the innermost region of disk: "dips in X-ray emission are followed by ejections of bright superluminal knots in the radio jet" (Marscher et al. 2002). The detectability of the Fe K $\alpha$ line in radio-loud objects may also be influenced by contamination of the X-ray continuum by non-thermal (beamed) emission.

\subsection{Empirical evidence for a disk-jet connection}

The correlation between the extended radio and line emission has been explored by a number of authors (e.g., Baum \& Heckman 1989a,b; Rawlings \& Saunders 1991; Rawlings 1992; Falcke et al. 1995; Xu et al. 1999; Cao \& Jiang 1999; Willott et al. 1999). The present sample allows us not only to further test this correlation, but presents us with an opportunity to explain it in the context of our improved model. Figures $3 \mathrm{a}, \mathrm{b}$ show that the extended radio emission is evidently quite closely linked with $\lambda$ and $\Lambda$. The linear regression for the RLQs alone is

$\log L_{5 \mathrm{GHz}}^{\mathrm{ext}}=(28.05 \pm 0.78)+(0.95 \pm 0.38) \log \lambda$,

with a Pearson's correlation coefficient $r=0.56$, significant at the level of $p=3.8 \times 10^{-3}$ according to Student's $t$ test. Similarly,

$\log L_{5 \mathrm{GHz}}^{\mathrm{ext}}=(-8.65 \pm 11.03)+(1.45 \pm 0.46) \log \Lambda$,

with $r=0.71$ and $p=6.2 \times 10^{-5}$.

For comparison, we include the BL Lac objects studied by Wang et al. (2002) that have extended radio luminosities given by Urry et al. (2000); there are 12 high frequencypeaked (HBL) and 15 low frequency-peaked (LBL) objects. The BL Lac objects smoothly extend the sequence defined by the RLQs toward lower values of $L_{5 \mathrm{GHz}}, \lambda$, and $\Lambda$. The correlations are now enhanced, becoming

$\log L_{5 \mathrm{GHz}}^{\mathrm{ext}}=(27.57 \pm 0.29)+(0.68 \pm 0.10) \log \lambda$,

with $r=0.65$ and $p=2.6 \times 10^{-10}$, and

$\log L_{5 \mathrm{GHz}}^{\mathrm{ext}}=(4.18 \pm 2.70)+(0.92 \pm 0.11) \log \Lambda$,

with $r=0.76$ and $p=2.1 \times 10^{-15}$. In the above correlation analysis of $L_{5 \mathrm{GHz}}^{\text {ext }}$ versus $\lambda$, we excluded the upper limits for the BL Lac objects. A survival analysis including these upper limits shows $\log L_{5 \mathrm{GHz}}^{\mathrm{ext}}=(27.60 \pm 0.30)+(0.64 \pm 0.11) \log \lambda$ with Spearman's $\rho=0.667$. These correlations are striking in that they provide fairly direct evidence that the extended jet emission is somehow closely coupled to the central engine via the accretion disk. The most direct interpretation is that the extended radio emission is fed by relativistic jets that are tightly linked to the accretion disk (Rees 1984).

Following Meier (2001), we assume that $1 \%$ of the MHD energy will be radiated as radio emission with a spectral index of -1 . Then, for a Kerr $\mathrm{BH}$ and using the relation $\Lambda=\eta \xi \dot{M}$, $L_{5 \mathrm{GHz}}^{\mathrm{ext}}=3.74 \times 10^{26} \epsilon_{-2} m_{9} \dot{m}_{-1} \mathrm{~W} \mathrm{~Hz}^{-1}$, or

$L_{5 \mathrm{GHz}}^{\mathrm{ext}}=3.74 \times 10^{27} \epsilon_{-2} m_{9} \lambda \mathrm{W} \mathrm{Hz}^{-1}$

These expressions are in rough agreement with the observed correlations. 


\subsection{A power limit for radio-loud quasars?}

In our sample, only PKS 0454-22 is a super-Eddington accretor $\left(E_{R}=10^{0.7}\right)$. The most powerful RLQs, which have powers in excess of $10^{47} \mathrm{erg} \mathrm{s}^{-1}$, potentially may be super-Eddington accretors (Ghisellini \& Celotti 2001). In this section we consider the upper luminosity limit of RLQs as a threshold set by super-Eddington accretion.

For a $\mathrm{BH}$ accreting well above the Eddington rate, much of the dissipated energy will be advected into $\mathrm{BH}$ because most of the photons are trapped in the flow by Thomson scattering (Wang \& Zhou 1999; Ohsuga et al. 2002). Photon trapping efficiently controls the radiated luminosity; the radiated luminosity from the disk depends very weakly on the accretion rate, $L_{\text {disk }} \propto \log \dot{M}$, tending to be saturated. For a self-similar disk (Wang \& Zhou 1999), Eq. (15) reduces to

$a_{\mathrm{s}} \approx 0.65 r^{-0.5}$

and the MHD power is given by

$L_{\text {jet }}=8.05 \times 10^{46} \dot{m}_{1} m_{9} \mathrm{erg} \mathrm{s}^{-1}$

for a Schwarzschild $\mathrm{BH}$ and

$L_{\text {jet }}=3.76 \times 10^{47} \dot{m}_{1} m_{9} \mathrm{erg} \mathrm{s}^{-1}$

for a Kerr BH, where $\dot{m}_{1}=\dot{m} / 10$. The power is still proportional to the accretion rate, although the radiation from disk itself is saturated. In the super-Eddington regime, the difference in radio power between a Kerr and Schwarzschild $\mathrm{BH}$ is smaller.

From the total rotational energy due to the spin, $E_{\text {spin }} \approx$ $1.6 \times 10^{62} m_{9} j^{2} \mathrm{erg}$, it follows that the spin-down timescale due to the $\mathrm{BZ}$ process is

$\tau_{\text {spin-down }}=1.9 \times 10^{7} \dot{m}_{1}^{-1} \mathrm{yr}$.

This is much shorter than a typical merger timescale of $10^{9} \mathrm{yr}$ (Wilson \& Colbert 1995), if the spin angular momentum comes from galaxy collisions. If the powerful jet is fed by a Schwarzschild $\mathrm{BH}$ via super-Eddington accretion, the $e$-folding timescale of the $\mathrm{BH}$ growth will be

$\tau_{\mathrm{SEA}}=M_{\mathrm{BH}} / \dot{M}=4.0 \times 10^{7} \dot{m}_{1}^{-1} \mathrm{yr}$.

The lifetime of the powerful jet from an SEA is rather short unless there is continuous super-Eddington fueling. Thus, superEddington RLQs ought to be very rare.

\subsection{AGN unification}

A useful diagnostic for radio galaxies, as first shown by Owen \& Ledlow (1994; see also Ledlow \& Owen 1996 and Owen et al. 1996), is the plot of extended radio luminosity versus optical absolute magnitude. A diagonal line well separates the two radio populations: at a given optical luminosity (which in the case of radio galaxies is dominated by the host galaxy), FR IIs have much higher radio powers than FR Is (Fanaroff $\&$ Riley 1974). The physical interpretation of the OwenLedlow line has been much debated. Bicknell (1995) and
Gopal-Krishna \& Wiita (2001) suggest that it may arise naturally from the interaction of the jet with the interstellar or intracluster medium. Or perhaps the two classes reflect intrinsic differences in their central engines, such as BH spin (Baum et al. 1995; Meier 1999) or state of the accretion disk. Ghisellini \& Celotti (2001) recently suggested that the dividing line corresponds to a transition in the accretion mode, from an SS disk (FR II) to an optically thin ADAF (FR I).

Urry et al. (2000) also examined BL Lac objects and RLQs in the $M_{R}-L_{\text {radio }}^{\text {ext }}$ diagram, but the data for their RLQs are mostly not from $H S T$ observations. Figure $3 \mathrm{c}$ gives a new representation of the Owen-Ledlow diagram for RLQs and BL Lacs, where we have substituted the optical luminosity on the abscissa with $\mathrm{BH}$ mass $M_{\mathrm{BH}}$. We have plotted our sample of RLQs along with the sample of BL Lac objects studied by Urry et al. (2000). The diagonal line is the Owen \& Ledlow (1994) line for radio galaxies, adapted from Ledlow \& Owen (1996) after converting to our distance scale, the radio luminosities from $1.4 \mathrm{GHz}$ to $5 \mathrm{GHz}$ assuming a spectral index of -1 , and $M_{R}$ to $M_{\mathrm{BH}}$ using Eq. (1).

Several features are noteworthy in this diagram. First, the Owen-Ledlow line that divides FR I and FR II radio galaxies seems to cleanly bisect the population of beamed radioloud objects. Nearly all HBLs fall below the line, and all but two FSRQ lies above the line. Second, the population of LBLs straddle the divide, occupying the region between HBLs and FSRQs. Third, as with the FSRQs, essentially all SSRQs lie above the line. And fourth, at least some BL Lac objects, mostly of the LBL variety, appear to be more closely associated with FR II radio galaxies; this is in agreement with the suggestion of Kollgaard et al. (1996) that some radio-selected BL Lac objects may be beamed FR II sources.

The availability of accretion rates enables us to consider radio-loud AGNs in a more unified, physical context. Wang et al. (2002) show that most BL Lac objects have accretion rates below the critical value $\dot{m} \approx \alpha^{2}$ that demarcates the transition from a standard SS disk to an optically thin ADAF. Moreover, they find that HBLs have characteristically lower accretion rates than LBLs. This study demonstrates that nearly all RLQs have $\dot{m}>\alpha^{2}$. It is important to note that, at least for the sample considered here, most of the sources occupy a relatively narrow range of $\mathrm{BH}$ masses, $\sim 10^{8.5} M_{\odot}$ with a spread of $\sim 0.5 \mathrm{dex}$. The various subpopulations of radio-loud AGNs can therefore be cast into a physical, perhaps evolutionary, sequence described by a single variable - accretion rate. For the beamed population, $\dot{m}$ decreases along the sequence FSRQ $\rightarrow$ $\mathrm{LBL} \rightarrow \mathrm{HBL}$. When viewed at large angles to our line of sight, the sequence becomes FR II $\rightarrow$ (FR II or FR I) $\rightarrow$ FR I. Since the structure of the accretion flow is governed by $\dot{m}$, the variation in $\dot{m}$ along the sequence directly translates into a variation in the dominant mode of accretion. The most powerful RLQs and FR II radio galaxies may contain super-Eddington disks or optically thick ADAFs. The majority of RLQs and FR IIs have SS disks. The low-power HBLs and some LBLs have pure optically thin ADAFs. Objects that occupy the fuzzy boundary near the Owen-Ledlow line, which comprise some LBLs and a minority of FR IIs, may have a hydrid structure consisting of an SS disk plus an optically thin ADAF. 
The basic elements of the above evolutionary scenario, based on complementary but different lines of evidence, have already been advocated by a number of authors (e.g., Ghisellini et al. 1998; Ghisellini \& Celotti 2001, 2002; Ghisellini et al. 2002; Böttcher \& Dermer 2002; Cavaliere \& D'Elia 2002). Böttcher \& Dermer (2002) and Cavaliere \& D'Elia (2002) argue that RLQs evolve into BL Lacs rather than BL Lacs into RLQs (Böttcher \& Dermer 2002). If the evolutionary direction is from RLQs to BL Lacs, the BH masses of RLQS should be systematically smaller than those of BL Lacs. However, the significant overlap in the $\mathrm{BH}$ mass distributions of the two groups (Fig. 3c; see also Falomo 2003) does not seem to support this single evolutionary direction. Given the current uncertainties in $\mathrm{BH}$ mass determinations for AGNs, it is probably premature to draw strong conclusions. Here, we merely wish to note that both evolutionary paths can, and probably do, occur. The detection of quasar remnants in nearby galaxies in the form of inactive massive BHs conclusively demonstrates that the cosmic evolution of AGN activity must be episodic (Richstone et al. 1998). The duty cycle for accretion is short, such that any individual massive $\mathrm{BH}$ is likely to have been activated (and deactivated) many times since it was formed.

\section{Conclusions}

We attempt to constrain the accretion rates and the mode of accretion in RLQs using BH masses estimated from HST imaging of their host galaxies and accretion luminosities derived from their emission-line spectra. The quasars have large $\mathrm{BH}$ masses, $M_{\mathrm{BH}} \approx 10^{8}-10^{9} M_{\odot}$, and substantial accretion rates, $\dot{M} \approx$ 0.01-1 times the Eddington rate. Their accretion rates suggest that most of the objects possess standard optically thick, geometrically thin accretion disks, perhaps in combination with an optically thin ADAF in some cases. Our sample has a deficit of objects with super-Eddington disks, although this is likely due to a selection effect in the sample.

Our data can also be used to test current ideas on jet formation. We present an improved model for a standard optically thick, geometrically thin disk that incorporates the effects of advection cooling. Our model can explain the level of radio emission observed in RLQs. We discuss some consequences of our model for the interpretation of $\mathrm{Fe} \mathrm{K} \alpha$ lines observed in the $\mathrm{X}$-rays.

Finally, we combine the present sample of RLQs with a recently analyzed sample of BL Lac objects to reevaluate the unification picture for radio-loud AGNs. The strong correlation found between the luminosity of the extended radio emission and the accretion rate supports the notion that radio jets are directly coupled to the accretion disk. In agreement with prevailing ideas, our results support the proposition that FSRQs and BL Lacs are the beamed counterparts of FR II and FR I radio galaxies, respectively. However, we argue that some fraction of the low frequency-peaked BL Lac sources in fact may be more closely associated with FR II radio galaxies. The various subclasses of radio-loud AGNs - ranging from high-luminosity sources (FSRQs and FR IIs) on the one end to low-luminosity sources (BL Lacs and FR Is) on the other - can be viewed as a continuous sequence of varying accretion rate.
Acknowledgements. The authors are grateful to the referee for helpful comments and suggestions. J. M. W. thanks D. L. Meier for useful discussions. He is grateful to the support from Alexander von Humboldt Foundation, the Hundred Talent Program of Chinese Academy of Sciences, and the Special Funds for Major State Basic Research Projects and NSFC. The research of L. C. H. is supported by the Carnegie Institution of Washington and by NASA grants from the Space Telescope Science Institute (operated by AURA, Inc., under NASA contract NAS5-26555).

\section{References}

Abramowicz, M. A., Czerny, B., Lasota, J. P., \& Szuszkiewicz, E. 1988, ApJ, 322, 646

Aldcroft, T. L., Elvis, M., \& Bechtold, J. 1993, AJ, 105, 2054

Bahcall, J. N., Kirhakos, S., \& Saxe, D. H. 1997, ApJ, 479, 642

Baldwin, J. A. 1975, ApJ, 201, 26

Baum, A. S., \& Heckman, T. M. 1989a, ApJ, 336, 681

Baum, A. S., \& Heckman, T. M. 1989b, ApJ, 336, 702

Baum, S. A., Zirbel, E. L., \& O’Dea, C. P. 1995, ApJ, 451, 88

Begelman, M. C. 2002, ApJ, 568, L97

Begelman, M., \& Meier, D. L. 1982, ApJ, 253, 873

Bergeron, J., \& Kunth, D. 1984, MNRAS, 207, 263

Bicknell, G. V. 1995, ApJS, 101, 297

Blandford, R. 2002, Progress of Theoretical Physics Supplement, in press [astro-ph/0110394]

Blandford, R., \& Begelman, M. C. 1999, MNRAS, 303, L1

Blandford, R. D., \& Payne, J. 1982, MNRAS, 199, 883

Blandford, R. D., \& Znajek, R. L. 1977, MNRAS, 179, 433

Bogers, W. J., Hes, R., Barthel, P. D., \& Zensus, J. A. 1994, A\&AS, 105,91

Boroson, T. A., \& Green, R. F. 1992, ApJS, 80, 109

Boroson, T. A., Persson, S. E., \& Oke, J. B. 1985, ApJ, 293, 120

Böttcher, M., \& Dermer, C. D. 2002, ApJ, 564, 86

Boyce, P., Disney, M. J., Blades, J. C., et al. 1998, MNRAS, 298, 121

Boyce, P., Disney, M., \& Bleaken, D. 1999, MNRAS, 302, L39

Brocksopp, C., Fender, R. P., Larionov, V., et al. 1999, MNRAS, 309, 1063

Brotherton, M. S. 1996, ApJS, 102, 1

Cao, X., \& Jiang, D. R. 1999, MNRAS, 307, 802

Cavaliere, A., \& D'Elia, V. 2002, ApJ, 571, 226

Celotti, A., \& Fabian, A. C. 1993, MNRAS, 264, 228

Celotti, A. C., Padovani, P., \& Ghisellini, G. 1997, MNRAS, 286, 415

Chen, X. 1995, ApJ, 448, 803

Chen, X., Abramowicz, M. C., Lasota, J.-P., Narayan, R., \& Yi, I. 1995, ApJ, 443, L61

Chen, X., \& Taam, R. 1993, ApJ, 412, 254

Corbin, M. R. 1992, ApJ, 391, 577

Corbin, M. R. 1997, ApJS, 113, 245

Danziger, I. J., \& Goss, W. M. 1983, MNRAS, 202, 703

Douglas, J. N., Bash, F. N., Bozyan, A., Torrence, G. W., \& Wolfe, C. 1996, AJ, 111, 1945

Dunlop, J. S., McLure, R. J., Kukula, M. J., et al. 2003, MNRAS, 340, 1095

Eracleous, M., \& Halpern, J. P. 1994, ApJS, 90, 1

Falcke, H., Malkan, M. A., \& Biermann, P. L. 1995, A\&A, 336, 702

Fanaroff, B. L., \& Riley, J. M. 1974, MNRAS, 167, 31P

Falomo, R. 2003, in High-Energy Blazar Astronomy, ed. A. Sillanpää, L. O. Takalo, \& E. Valtaoja (San Francisco: ASP), in press

Fender, R. P., et al. 1999, ApJ, 519, L165

Francis, P. J., Hewett, P. C., Foltz, C. B., et al. 1991, ApJ, 373, 465

Frank, J., King, A. R., \& Raine, D. J. 1992, Accretion Power in Astrophysics (Cambridge: Cambridge Univ. Press) 
Fukugita, M., Shimasaku, K., \& Ichikawa, T. 1995, PASP, 107, 945 Ghisellini, G., \& Celotti, A. 2001, A\&A, 379, L1

Ghisellini, G., \& Celotti, A. 2002, in Issues in Unification of AGNs, ed. R. Maiolino, A. Marconi, \& N. Nagar (San Francisco: ASP), 273

Ghisellini, G., Celotti, A., \& Costamante, L. 2002, A\&A, 386, 833

Ghisellini, G., Celotti, A., Fossati, G., Maraschi, L., \& Comastri, A. 1998, MNRAS, 301, 451

Ghisellini, G., Padovani, P., Celotti, A., \& Maraschi, L. 1993, ApJ, 407, 65

Ghosh, P., \& Abramowicz, M. A. 1997, MNRAS, 292, 887

Gondhalekar, P. M. 1990, MNRAS, 243, 443

Gopal-Krishna, \& Wiita, P. 2001, A\&A, 373, 100

Grandi, S. A., \& Phillips, M. M. 1979, ApJ, 232, 659

Gregory, P. C., \& Condon, J. J. 1991, ApJS, 75, 1011

Gu, W.-M., \& Lu, J.-F. 2000, ApJ, 540, L33

Hamilton, T. S., Casertano, S., \& Turnshek, D. A. 2002, ApJ, 576, 61

Harmon, B. A., Wilson, C. A., Zhang, S. N., et al. 1995, Nature, 374, 703

Harmon, B. A., Deal, K. J., Paciesas, W. S., et al. 1997, ApJ, 477, L85

Ho, L. C. 1999, in Observational Evidence for Black Holes in the Universe, ed. S. K. Chakrabarti (Dordrecht: Kluwer), 157

Ho, L. C. 2002a, in Issues in Unification of AGNs, ed. R. Maiolino, A. Marconi, \& N. Nagar (San Francisco: ASP), 165

Ho, L. C. 2002b, ApJ, 564, 120

Ho, L. C., Rudnick, G., Rix, H.-W., et al. 2000, ApJ, 541, 120

Hooper, E. J., Impey, C. D., \& Foltz, C. B. 1997, ApJ, 480, L95

Hooper, E. J., Impey, C. D., Foltz, C. B., \& Hewett, P. C. 1996, ApJ, 473, 746

Hutchings, J. B., Gower, A. C., Reneveld, S., \& Dewey, A. 1996, AJ, 111,2167

Hutchings, J. B., Holtzman, J., Sparks, W. B., et al. 1994, ApJ, 429, L1

Iwasawa, K., \& Taniguchi, Y. 1993, ApJ, 413, L15

Jackson, N., \& Browne, I. W. A. 1991, MNRAS, 250, 414

Kellermann, K. I., Sramek, R., Schmidt, M., Shaffer, D. B., \& Green, R. 1989, AJ, 98, 1195

Kollgaard, R. I., Palma, C., Laurent-Muehleisen, S. A., \& Feigelson, E. D. 1996, ApJ, 465, 115

Kormendy, J., \& Gebhardt, K. 2001, in The 20th Texas Symposium on Relativistic Astrophysics, ed. H. Martel, \& J. C. Wheeler (New York: AIP), 363

Kormendy, J., \& Richstone, D. 1995, ARA\&A, 33, 581

Lacy, M., Laurent-Muehleisen, S. A., Ridgway, S. E., Becker, R. H., \& White, R. L. 2001, ApJ, 551, L17

Ledlow, M. J., \& Owen, F. N. 1996, AJ, 112, 9

Lister, M. L., Gower, G. A., \& Hutchings, J. B. 1994, AJ, 108, 821

Liu, B. F., Yuan, W., Meyer, F., Meyer-Hofmeister, E., \& Xie, G. Z. 1999, ApJ, 527, L17

Livio, M., Ogilvie, G. I., \& Pringle, J. E. 1999, ApJ, 512, 100

Mahadevan, R. 1997, ApJ, 477, 585

Magorrian, J., Tremaine, S., Richstone, D., et al. 1998, AJ, 115, 2285

Malkan, M. A., \& Sargent, W. L. W. 1982, ApJ, 254, 22

Marscher, A. P., Jorstad, S. G., Gómez, J.-L., et al. 2002, Nature, 417, 625

Maraschi, L., \& Tavecchio, F. 2003, ApJ, 593, 667

Marziani, P., Sulentic, J. W., Dultzin-Hacyan, D., Calvani, M., \& Moles, M. 1996, ApJS, 104, 37

McCollough, M. L., Robinson, C. R., Zhang, S. N., et al. 1999, ApJ, 517,951

McLure, R. J., \& Dunlop, J. S. 2001, MNRAS, 327, 199

McLure, R. J., \& Dunlop, J. S. 2002, MNRAS, 331, 795

McLure, R. J., Dunlop, J. S., Kukula, M. J., et al. 1999, MNRAS, 308, 377
Meier, D. L. 1999, ApJ, 522, 753

Meier, D. L. 2001, ApJ, 548, L9

Nandra, K., George, I. M., Mushotzky, R. F., Turner, T. J., \& Yaqoob, T. 1997, ApJ, 476, 70

Narayan, R., Igumenshchev, I., \& Abramowicz, M. A. 2000, ApJ, 539, 798

Narayan, R., Mahadevan, R., \& Quataert, E. 1998, in The Theory of Black Hole Accretion Discs, ed. M. A. Abramowicz, G. Björnsson, \& J. E. Pringle (Cambridge: Cambridge Univ. Press), 148

Narayan, R., \& Yi, I. 1994, ApJ, 428, L13

Netzer, H. 1990, in Active Galactic Nuclei, ed. R. D. Blandford, H. Netzer, \& L. Woltjer (Berlin: Springer), 57

Neugebauer, G., Oke, J. B., Becklin, E. E., \& Matthews, K. 1979, ApJ, 230, 79

Ohsuga, K., Mineshige, S., Mori, M., \& Umemura, M. 2002, ApJ, 574,315

Oke, J. B., Shields, G. A., \& Korycanski, D. G. 1984, ApJ, 277, 64

Orr, M. J. L., \& Browne, I. W. A. 1982, MNRAS, 200, 1067

Osmer, P. S., Porter, A. C., \& Green, R. F. 1994, ApJ, 435, 678

Owen, F. N., \& Ledlow, M. J. 1994, in The Physics of Active Galaxies, ed. G. V. Bicknell, M. A. Dopita, \& P. J. Quinn (San Francisco: ASP), 139

Owen, F. N., Ledlow, M. J., \& Keel, W. C. 1996, AJ, 111, 53

Phillips, M. M. 1978, ApJS, 38, 187

Quataert, E., Di Matteo, T., Narayan, R., \& Ho, L. C. 1999, ApJ, 525, L89

Quataert, E., \& Gruzinov, A. 2000, ApJ, 539, 809

Rawlings, S. 1992, in Extragalactic Radio Sources. From Beams to Jets, ed. J. Roland, H. Sol, \& G. Pelletier (Cambridge: Cambridge Univ. Press), 332

Rawlings, S., \& Saunders, R. D. E. 1991, Nature, 349, 138

Rector, T. A., Stocke, J., \& Ellingson, E. 1995, AJ, 110, 1492

Rees, M. J. 1984, ARA\&A, 22, 471

Reeves, J. N., \& Turner, M. J. L. 2000, MNRAS, 316, 234

Richstone, D. O., Ajhar, E. A., Bender, R., et al. 1998, Nature, 395, A14

Rózanska, A., \& Czerny, B. 2000, A\&A, 316, 473

Sambruna, R., Eracleous, M., \& Mushotzky, R. F. 1999, ApJ, 526, 60

Scarpa, R., \& Falomo, R. 1997, A\&A, 325, 109

Shakura, N. I., \& Sunyaev, R. A. 1973, A\&A, 24, 337

Shields, G. A. 1978, Nature, 272, 706

Stockton, A., \& MacKenty, J. W. 1987, ApJ, 316, 584

Urry, C. M., \& Padovani, P. 1995, PASP, 107, 803

Urry, C. M., Scarpa, R., O’Dowd, M., et al. 2000, ApJ, 532, 816

Wang, J.-M. 2003, AJ, 125, 2859

Wang, J.-M., Staubert, R., \& Ho, L. C. 2002, ApJ, 579, 554

Wang, J.-M., Szuszkiewicz, E., Lu, F.-J., \& Zhou, Y.-Y. 1999, ApJ, 522,839

Wang, J.-M., \& Zhou, Y.-Y. 1999, ApJ, 516, 420

White, M. 1992, ApJS, 79, 49

Wilkes, B. J., Kuraszkiewicz, J., Green, P. J., Mathur, S., \& McDowell, J. C. 1999, ApJ, 513, 76

Willott, C. J., Rawlings, S., Blundell, K. M., \& Lacy, M. 1999, MNRAS, 309, 1017

Wills, B. J., Thompson, K. L., Han, M., et al. 1995, ApJ, 447, 139

Wilson, A. S., \& Colbert, E. J. M. 1995, ApJ, 438, 62

Xu, C., Livio, M., \& Baum, S. 1999, AJ, 118, 1169

Zheng, W., Kriss, G. A., Telfer, R. C., Grimes, J. P., \& Davidsen, A. F. 1997, ApJ, 475, 469 (Erratum: 1998, 492, 855) 